\title{
Impact of High-Speed Image Recognition of Transition Phenomenon of Chip Formation and Chip Flow in Gear Hobbing Process
}

\author{
Yoji Umezaki ${ }^{a}$, Syuhei Kurokawa ${ }^{\mathrm{b}}$ and Yasutsune Ariura ${ }^{\mathrm{c}}$ \\ Department of Mechanical Engineering, Graduate School of Engineering, \\ Kyushu University \\ 744, Motooka, Nishi-ku, Fukuoka, 819-0395, Japan \\ a umezaki@mech.kyushu-u.ac.jp, ${ }^{b}$ kurobe-@mech.kyushu-u.ac.jp, \\ ${ }^{c}$ ariurtde@mbox.nc.kyushu-u.ac.jp
}

\begin{abstract}
Keywords: High-speed Image Recognition, Gear, Chip Flow Measurement, Hobbing, Chip Formation, Chip Interference
\end{abstract}

\begin{abstract}
The transient phenomenon of chip generations and behavior in the gear hobbing process are investigated by using a high-speed video camera. The chip behavior generated in gear finish hobbing process is very complicated and one can not identify each chip from specified cutting edges. The authors have built up a new simulation method of the hobbing process using a flying tool and a special-shaped workpiece, which consists of one tooth space. Visual evidences of the chip interference on the rake face and some conditions of contact between generated chips and the work surface were visually obtained. In the case of dry cutting conditon with a high-speed steel (HSS) flytool without coating on the rake face, the flytool cuts the workpiece frequently with the stuck chip generated in the previous revolution on the rake face. The newly generated chip pushes out the previous stuck chip, which flies away eventually. The chip flow on the rake face interferes strongly at the corner of the cutting edge when both top and side cutting edges produce different chips at the same time, and the chips flow out in changing the shape. The moving speed of the chip was also measured.
\end{abstract}

\section{Introduction}

Gear hobbing is one of the most complicated and severest cutting processes, which has peculiar characteristics of intermittent cutting with multi-cutting edges, producing different-shaped chips and having different situations of wear in every cutting edge, and being applicable in dry cutting conditions. However, transition phenomenon of the hobbing process could not be observed yet due to high-speed phenomenon and being blocked out of sight by adjacent cutting edges. Observation of the chip generation and the chip flow has been a long-cherished dream among gear people. Up to now, cutting transition information is estimated only by observation of cut-away chips and tool wear after hobbing. Chips show that there must be strong interference during chip flow in each cutting edge and the interference is regarded as one of the reasons of tool wear due to severe stress on the tool surface.

On the other hand, observation of transition phenomenon in simple cutting process was reported in the past by using the image recognition in turning process for example [1]. In addition, appearance of a high-speed camera enables us to observe the high-speed phenomenon in recent years. Nevertheless, it is impossible to observe the transition phenomenon in the hobbing process because the target is blocked out of sight by adjacent cutting edges and it is the fate for the rotating multi-edge cutting tools. So nobody has caught the direct evidence in chip generation.

The authors have developed a new testing method on a hobbing machine to simulate hobbing mechanisms by a flytool [2]. A special-shaped gear blank is prepared with the help of the numerical hobbing simulator [3] developed by the authors. Using a high-speed video camera, the chip generation and the chip flow on the rake face of the flytool was successfully observed directly by high-speed image recognition for the first time. The interference during chip flow was also 
observed and the moving speed of the chip on the rake face was measured by image processing. Discussions are also given on the chip interference, the adhesion of the chip on the rake face, and the pressure bonding phenomenon among successive chips.

\section{Observation of the Chip Generation in the Normal Gear Hobbing Process}

Gear hobbing using triple thread HSS hob without coating was observed with a high-speed video camera. A special workpiece which has the shape of a spur gear tooth space was prepared. The cutting test was performed under the conditions of the cutting speed of $80 \mathrm{~m} / \mathrm{min}$, the feed rate of $1.5 \mathrm{~mm} / \mathrm{rev}$, and the dry cutting.

The high-speed video camera (Redlake Motion Scope PCI) was set at the outside of the hobbing machine (Fig. 1) because there was no space inside of the hobbing machine, and because the accumulation of chips on the camera lens should have been avoided. Indirect images on a mirror mounted under the workpiece were captured to observe the chip generation and movements on the rake face of each hob cutting tooth. Sequential images were captured at 1000 frames per second (fps) and 1/3000 second exposure.

Figure 2 shows one frame of captured images during chip generation in the hobbing test. The workpiece and the hob rake face can be observed, but the following difficulties have arisen: (1) the target cutting edge is blocked out of sight by the foregoing cutting tooth (2) it is difficult to identify the chip from the specified cutting edge. In order to solve those difficulties, the authors have conceived the new testing method using single flytool so called flytool hobbing in this paper.

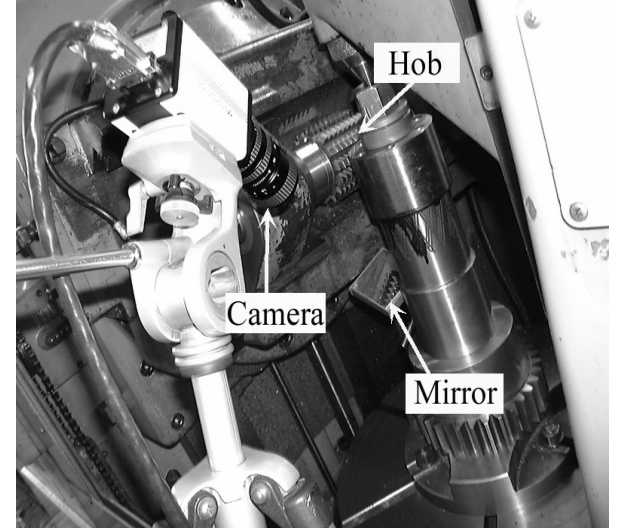

Fig. 1. Measuring system with the hobbing machine

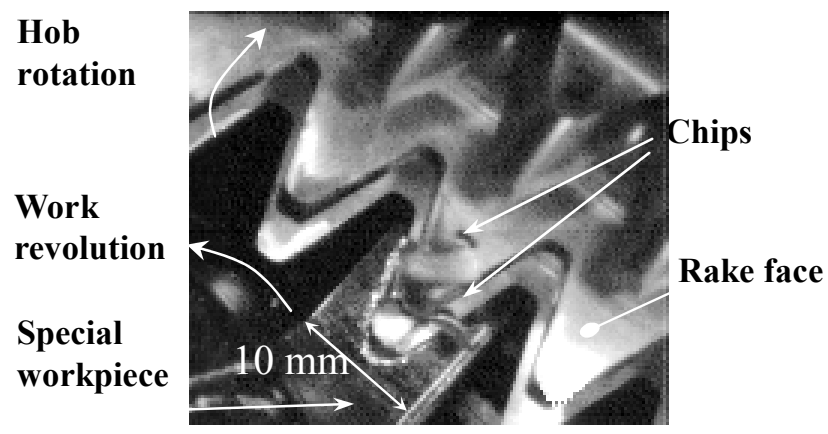

Fig. 2. Chip generation in the hobbing process

\section{New Flytool Hobbing Method}

The flytool hobbing method utilizes single flytool and a special-shaped workpiece which consists of one gear tooth space. The flytool used in this test has the same shape as the hob cutting tooth, and is mounted on the hobbing machine. The simulation of the hobbing process can be carried out by the intermittent cutting with single flytool.

The flytool is set at first at the location of the most preceding cutting edge which cuts the tooth space firstly, and after cutting along the tooth width, it is shifted in the next place and is rotated to the location of the next hob cutting edge in free generation [2]. Thus, single flytool generates the special-shaped gear tooth space instead of many hob cutting edges from one end in the roughing zone to the generation zone.

Only one kind of cutting is done during one feed along the tooth width in the flytool hobbing, while many kinds of cutting are occurred in the normal hobbing process. The numerical analysis method by Terashima and Ueno [3] was applied to this flytool hobbing analysis. One groove is 
formed firstly in the tooth space. The next groove is formed by the next located flytool movement and those groove formations are repeated, resulting in final gear tooth space.

Figure 3 shows the tooth space shapes after cutting by the second preceding cutting tooth from the central edge in both cases of the normal gear hobbing with triple thread hob and the flytool hobbing. Solid lines in Fig. 3 indicate tooth space shapes in different cross sections along the tooth width and dashed lines indicate the cutting zones by the second preceding cutting tooth (No.-2 cutting tooth) in the corresponding cross sections.

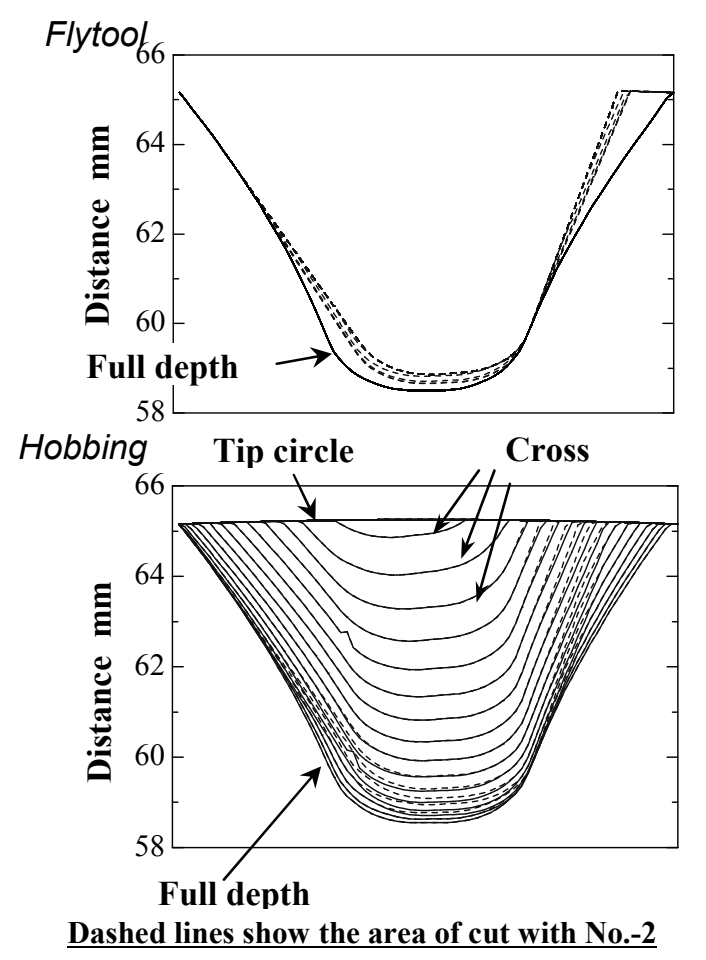

Fig. 3. Calculated tooth space shapes

In the normal gear hobbing, each cutting zone is wide (large working angle of a hob tooth) and it has long cutting length. On the other hand, in the flytool hobbing, the No.-2 flytool starts to cut deeply into the tooth space, because the large portion of the tooth space is already removed by the preceding flytool edges, resulting in the short cutting length. The cut volume per one pass of the cutting tooth in the gear hobbing is larger than that in the flytool hobbing to some extent. However the metal removal is not occurred at the corner between the round cutting edge and the side cutting edge in both cases. That means the characteristic of No.-2 cutting tooth is simulated well in the flytool hobbing.

Difference between the normal gear hobbing and the flytool hobbing is in the tooth space shape per one feed. Strictly speaking, some chip shapes between the gear hobbing and the flytool hobbing are not completely the same. However the cutting area on the cutting edge is similar with each other, and the chip shapes are almost the same. Therefore the flytool hobbing test is regarded as the good simulation test for the gear hobbing.

Excellent advantages in the flytool hobbing method are as follows:

(1) The flytool used in this test has the same geometrical shape as one hob cutting tooth, involving its relief angle and lead angle.

(2) The generating motion between the workpiece and the tool is realized by use of the actual hobbing machine. That means that completely the same generating motion can be achieved.

(3) One chip is formed per one cutting edge by using a special-shaped workpiece of one gear tooth space.

(4) Movements and behavior of the generated chip can be observed in detail without obstruction of the adjacent cutting teeth. 
The special-shaped workpieces were prepared to simulate the climb hobbing process of spur and helical gear teeth under the dry cutting condition in this paper.

\section{Chip Movements on the Rake Face in the Flytool Hobbing for Single Thread Hob}

The flytool hobbing test simulated for the spur with single thread hob typed flytool was performed under the conditions of the cutting speed of $80 \mathrm{~m} / \mathrm{min}$, the feed rate of $1.5 \mathrm{~mm} / \mathrm{rev}$, and the dry cutting. Chip movements by No.-16 cutting tooth and by No.-14 cutting tooth are shown in Fig. 4 and Fig. 5 respectively. One (Fig. 4) or two (Fig. 5) chips were already stuck on the rake face before the cut. The flytool starts to cut with the stuck chip and forms a new chip as protruding the previous stuck chip. In the case of No.-14 flytool, the new chip grows on the rake face, and only the first previous stuck chip is dispersed away.

From the results, there are many cases that the flytool without coating cuts with stuck chips on the rake faces in the next cutting. The jamming of the stuck chip on rake faces between finished surface and the cutting edge does not occur at least under the condition of $80 \mathrm{~m} / \mathrm{min}$ speed. The jamming of chips will be expected when an obstruction exists at the front of the flying chip after being protruded. The change of cutting states such as the shear angle, the friction angle and the contact length caused by the boundary of welding or compressed layer between the chip and the rake face will influence the tool wear and the jamming.
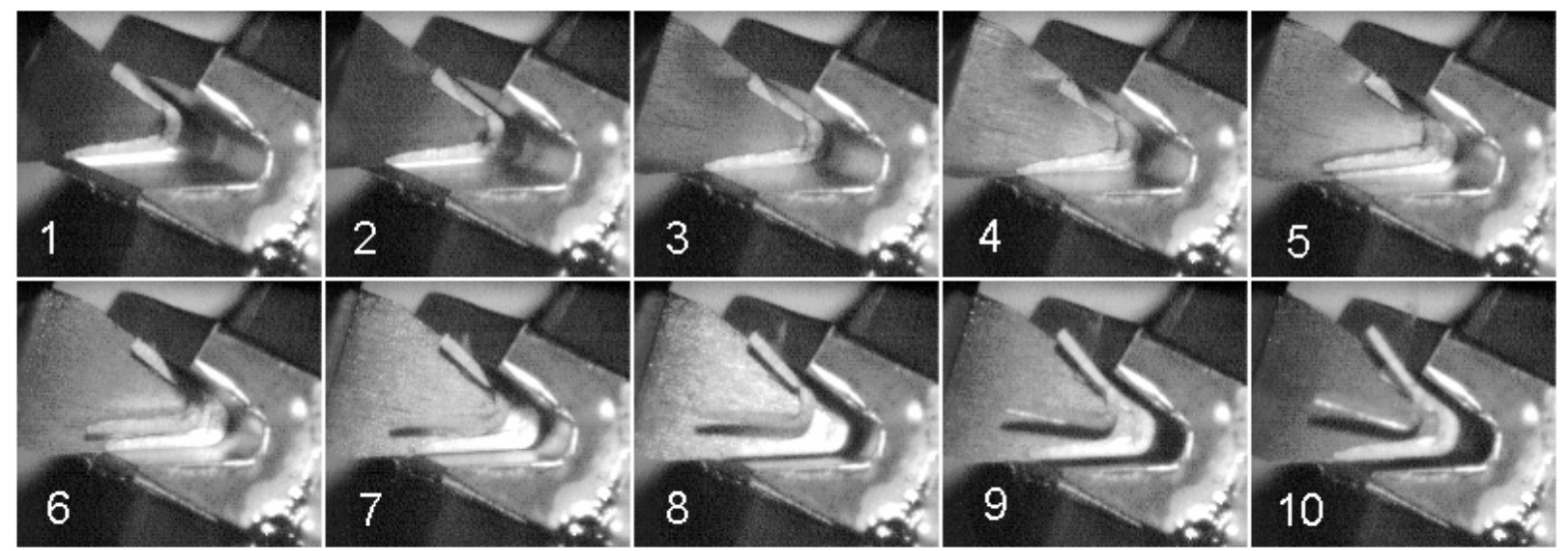

Fig. 4. High-speed images in the flytool hobbing with No.-16 cutting tooth
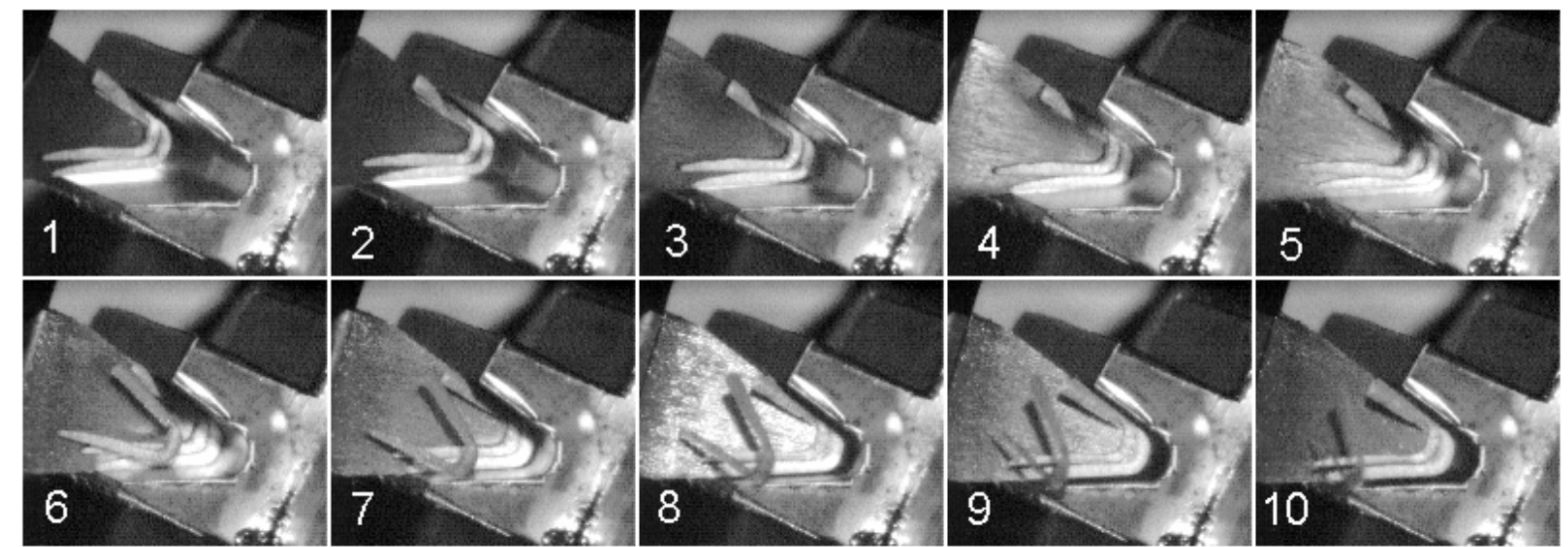

Fig. 5. High-speed images in the flytool hobbing with No.-14 cutting tooth 


\section{Chip Interference Observation}

In order to observe the chip interference directly, the experiment for the 20 degree helical gear with triple thread hob typed flytool was carried out under the conditions of the cutting speed of $80 \mathrm{~m} / \mathrm{min}$, the feed rate of $1.5 \mathrm{~mm} / \mathrm{rev}$, and the dry cutting. Figure 6 shows the chip generation with the No.-6 flytool captured at 4000 fps in every three frames. This test was done under the condition without stuck chips on the rake face by brushing. The interference between the chip from the top cutting edge and the chip from the leading side edge occurred firstly at the corner round part of the leading edge. The second interference started at No.5 frame, and severe interference was recognized after No.6 frame. The chips from both side cutting edges went under the chip from the top cutting edge, and the chip from the top cutting edge deformed and piled up at both round parts as the result of interference.

The chip flow speed was measured from the sequential images of the No. 2 frame and the frame near No.6 of Fig. 6. The distance between two adjacent pixels was about $0.0876 \mathrm{~mm}$, resulting in flowing speed of $478 \mathrm{~mm} / \mathrm{s}$ in the direction of the side flow. The rotational speed of flytool was about $1.33 \mathrm{~m} / \mathrm{s}(80 \mathrm{~m} / \mathrm{min})$ and the ratio of the rotational speed and the flowing speed becomes about 0.32 . This value is nearly equal to the cutting ratio.

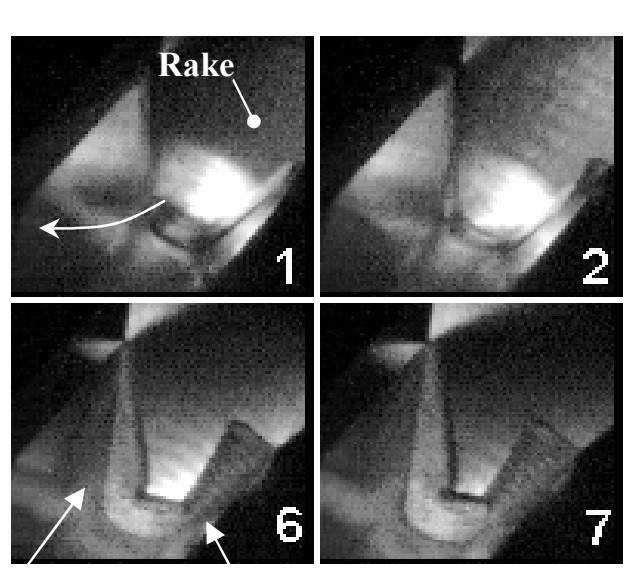

Leading side (A) Trailing side (B)

Fig. 6. High-sped images of the chip interference in the flytool hobbing Interference
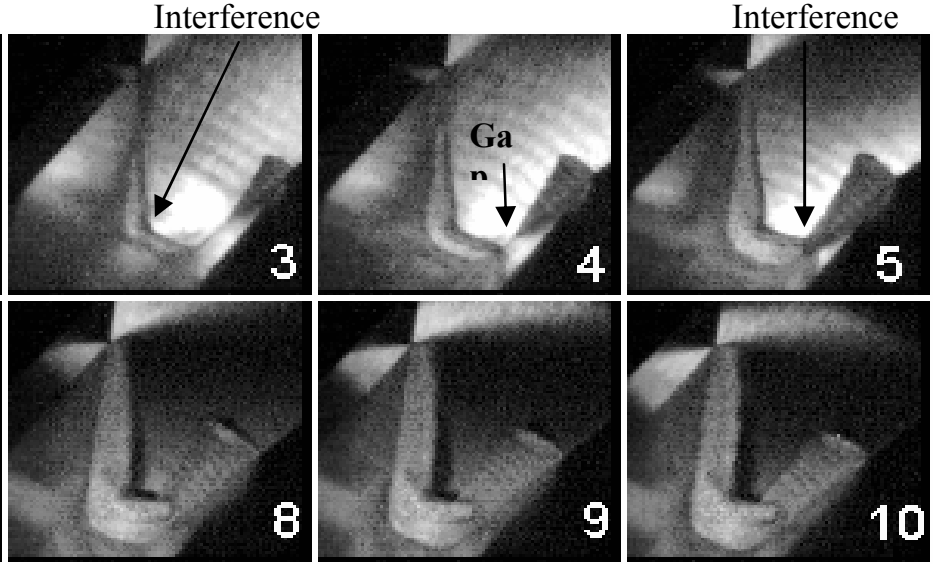

$1 \mathrm{~mm}$

$\longmapsto$ with No.-6 cutting tooth

\section{Conclusion}

Identification of transient phenomenon of the chip during generation in the gear hobbing by using a high-speed video camera and by the newly established flytool hobbing method was successfully performed for the first time.

The results obtained in the flytool hobbing test are as follows:

(1) The chip is stuck on the rake face and is protruded by the next generated chip in the case of non-coated rake face.

(2) The chip flows as deforming due to severe interference at the corner when the top and the side cutting edge produce the chip simultaneously.

\section{References}

[1] H.K. Tönshoff, et al.: Micro-cinematograph Investigations of Cutting Process, Film at Colloquium on Cutting of Metals, St. Etienne, France (1979).

[2] Y. Umezaki, et al.: Transient Phenomenon of Chip Generations and Movements in Hobbing, The JSME International Conference on Motion and Power Transmissions, MPT2001-Fukuoka, (2001), pp. 327-332.

[3] K. Terashima, T. Ueno: Numerical Analysis of Hobbing in Unfinished Space, Bulletin of the JSME, Vol.21, No.155 (1978), pp. 907-914. 